\title{
Caso 4/2006 - Mulher de 74 Anos com Cardiopatia Restritiva que Evoluiu para Choque Cardiogênico
}

\author{
Case 4/2006 - A 74 Year-old Woman Presenting Restrictive Cardiomyopathy and \\ Cardiogenic Schock
}

George Paulo Cobe Fonseca e Luiz Alberto Benvenuti Instituto do Coração do Hospital das Clínicas - FMUSP - São Paulo, SP

Mulher de 74 anos de idade foi trazida ao hospital em estado de confusão mental e com as extremidades frias.

A paciente apresentava há dois anos dispnéia desencadeada por esforços progressivamente menores, com piora há dois meses. Há duas semanas apresentava dispnéia mesmo em repouso e quando se deitava. Foi submetida a avaliação médica, que incluiu ecocardiograma (30.7.2003), o qual revelou disfunção sistólica acentuada de ventrículo esquerdo, insuficiência acentuada das valvas mitral e tricúspide e volumoso derrame pericárdico. Foram prescritos $50 \mathrm{mg}$ de captopril, $40 \mathrm{mg}$ de furosemida e $50 \mathrm{mg}$ de espironolactona diários. Havia também antecedente de evento isquêmico encefálico há um ano, sem seqüelas motoras.

Duas semanas depois dessa avaliação médica, apresentou rebaixamento do nível de consciência e foi trazida ao Hospital.

O exame físico (11.8.2003) revelou paciente torporosa, em mau estado geral, pulsos finos com 100 bpm e pressão arterial inaudível, palpada em $40 \mathrm{mmHg}$. A pressão venosa jugular estava discretamente elevada. Não havia déficit motor. A semiologia pulmonar foi normal, o exame do coração revelou sopros sistólicos mitral $+++/ 4+$ e tricúspide $++/ 4+$. O fígado foi palpado a $2 \mathrm{~cm}$ do rebordo costal direito e havia edema $++/ 4+$ em membros inferiores.

O eletrocardiograma (ECG) (11.8.2003) revelou freqüência cardíaca de $68 \mathrm{bpm}$, ritmo sinusal e escape ventricular - QRS largo, com morfologia de distúrbio de condução pelo fascículo ântero-superior do ramo esquerdo e anteriorização da alça de QRS (R puro em $V_{1}$ $\mathrm{e}_{2}$ ) (fig. 1). Alguns momentos depois, foram registrados episódios de taquicardia com freqüência de 137 bpm, com morfologia semelhante ao QRS do ECG inicial e ondas $\mathrm{P}$ dissociadas.

A glicemia capilar inicial foi de $38 \mathrm{mg} / \mathrm{dl}$; contudo, mesmo após a administração intravenosa de 40 g de glicose, não houve alteração do nível de consciência. A pressão arterial não se elevou após a administração rápida de solução de $\mathrm{NaCl}$ a $0,9 \%$. Foram iniciadas aminas vasoativas e realizada intubação orotraqueal para suporte respiratório.

Os exames laboratoriais são apresentados na tabela 1.

O ecocardiograma (11.8.2003) revelou espessuras de septo interventricular e de parede posterior de ventrículo esquerdo $14 \mathrm{~mm}$; diâmetro diastólico de ventrículo esquerdo $47 \mathrm{~mm}$, e diâmetro sistólico do ventrículo esquerdo 38 mm, fração de encurtamento 19\%. O diâmetro da aorta foi $32 \mathrm{~mm}$ e havia aumento acentuado do átrio esquerdo. 0 ventrículo direito era hipocinético e a pressão estimada da artéria pulmonar foi de $50 \mathrm{mmHg}$. Havia, ainda, calcificação de valva aórtica, sem estenose, derrame pericárdico discreto e derrame pleural esquerdo.

Foram administradas amiodarona, heparina (logo suspensa), dopamina, depois noradrenalina e furosemida por via intravenosa.

O ecocardiograma (13.8.2003) foi repetido e revelou espessura de septo interventricular, $15 \mathrm{~mm}$, de parede posterior de ventrículo esquerdo, $16 \mathrm{~mm}$; diâmetros de ventrículo esquerdo $40 \mathrm{~mm}$ (diastólico) e $36 \mathrm{~mm}$ sistólico; diâmetro de aorta de $29 \mathrm{~mm}$, aumento acentuado do átrio esquerdo, átrio direito e ventrículo direito, esse último com aumento da espessura das suas paredes. A valva mitral apresentava discreta insuficiência, enquanto a tricúspide tinha insuficiência intensa. 0 pericárdio era normal e havia derrame pleural à esquerda.

Apesar do uso continuado de aminas vasoativas, a paciente continuou em choque e oligúria e sob sedação contínua, e apresentou bradicardia seguida de assistolia, sem resposta às manobras de ressuscitação (13.8.2003).

Editor da Seção: Alfredo José Mansur (ajmansur@incor.usp.br) Editores Associados: Desidério Favarato (dclfavarato@incor.usp.br) Vera Demarchi Aiello (anpvera@incor.usp.br)

Correspondência: Alfredo José Mansur - InCor - Av. Dr. Enéas de Carvalho Aguiar, 44 - 05403-000 - São Paulo, SP E-mail: ajmansur@incor.usp.br 


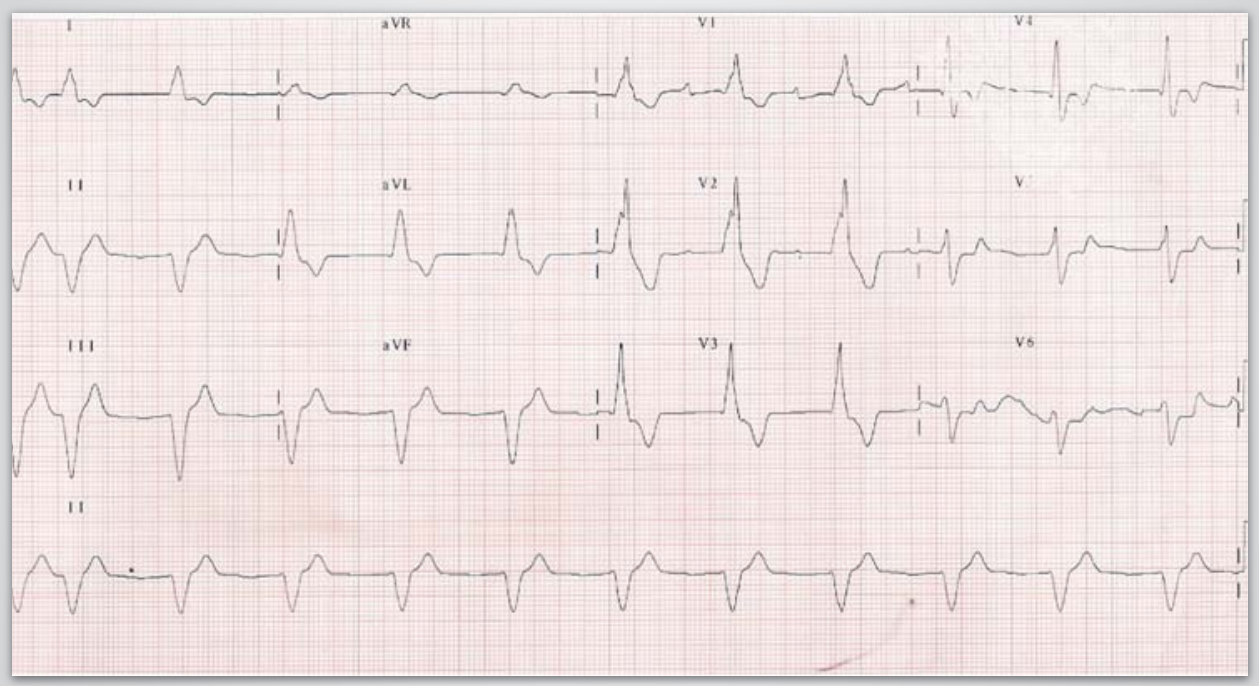

Fig. 1 - Eletrocardiograma. Ritmo sinusal e escape ventricular - QRS largo, com morfologia de distúrbio de condução pelo fascículo ântero-superior do ramo esquerdo e anteriorização da alça de $Q R S\left(R\right.$ puro em $V_{1}$ e $\left.V_{2}\right)$.

Tabela 1 - Exames laboratoriais

\begin{tabular}{|c|c|c|}
\hline Exame & 12.8.2003 & 13.8 .2003 \\
\hline Hemoglobina (g/dl) & 14,3 & 14,2 \\
\hline Hematócrito (\%) & 40 & 43 \\
\hline $\operatorname{VCM}\left(\mu \mathrm{m}^{3}\right)$ & 83 & 91 \\
\hline Leucócitos (/mm³) & 4900 & 5400 \\
\hline Neutrófilos (\%) & 85 & 81 \\
\hline Eosinófilos (\%) & 0 & 0 \\
\hline Basófilos (\%) & 0 & 0 \\
\hline Linfócitos (\%) & 7 & 16 \\
\hline Monócitos (\%) & 8 & 3 \\
\hline Plaquetas $/ \mathrm{mm}^{3}$ & 103000 & 98000 \\
\hline Sódio (mEq/l) & 120 & 129 \\
\hline Potássio (mEq/l) & 5,3 & 4,4 \\
\hline Magnésio (mEq/l) & 1,94 & - \\
\hline Creatinina (mg/dl) & 2,3 & 2,3 \\
\hline Uréia (mg/dl) & 178 & 179 \\
\hline Glicemia (mg/dl) & 148 & 90 \\
\hline Proteína total (g/dl) & 5,3 & - \\
\hline Albumina (g/dl) & 3,2 & - \\
\hline AST (U/I) & 52 & 30 \\
\hline $\operatorname{ALT}(\mathrm{U} / \mathrm{l})$ & 48 & 39 \\
\hline Gama GT (28 U/I) & 440 & 347 \\
\hline Amilase (100 U/I) & 194 & 142 \\
\hline Fosfatase alcalina (U/I) & - & 303 \\
\hline Bilirrubinas total (mg/dl) & 3,04 & 1,8 \\
\hline Bilirrubina direta (mg/dl) & 2,25 & 0,95 \\
\hline \multicolumn{3}{|l|}{ Gasimetria arterial } \\
\hline $\mathrm{FlO}_{2}(\%)$ & Masc $10 \mathrm{l} / \mathrm{min}$ & $30 \%$ \\
\hline $\mathrm{pH}$ & 7,26 & 7,47 \\
\hline $\mathrm{pCO}_{2}(\mathrm{~mm} \mathrm{Hg})$ & 27 & 24 \\
\hline $\mathrm{pO}_{2}(\mathrm{~mm} \mathrm{Hg})$ & 78 & 147 \\
\hline Sat. $\mathrm{O}_{2}(\%)$ & 94 & 99 \\
\hline $\mathrm{HCO}_{3}(\mathrm{mEq} / \mathrm{l})$ & 12 & 17 \\
\hline Excesso de bases $(\mathrm{mEq} / \mathrm{l})$ & -14 & $-4,4$ \\
\hline Lactato (mg/dl) & & 14 \\
\hline Dímero D (ng/ml) & & - \\
\hline $\mathrm{TSH}(\mu \mathrm{Ul} / \mathrm{ml})$ & & - \\
\hline T4 livre (ng/dl) & & \\
\hline TP (INR) & & 2,56 \\
\hline TTPA (paciente/controle) & & 1,24 \\
\hline
\end{tabular}




\section{Aspectos clínicos}

A paciente em referência já apresentava sintomas sugestivos da síndrome da insuficiência cardíaca esquerda havia pelo menos dois anos. Esses sintomas progressivamente tornaram-se mais intensos, até que duas semanas antes da internação hospitalar evoluíram para dispnéia em repouso e ortopnéia, ou seja, insuficiência cardíaca em classe funcional III/IV da NYHA.

A insuficiência cardíaca é a via final de várias doenças cardíacas, sendo as mais comuns a doença arterial coronariana, a hipertensão arterial sistêmica, a miocardiopatia dilatada não-isquêmica e a doença cardíaca valvar.

A insuficiência cardíaca é uma desordem relativamente prevalente, afetando mais de 4,6 milhões de pacientes nos Estados Unidos, e 550.000 casos novos por ano ${ }^{1}$. No Brasil, segundo o Datasus, existem atualmente 6,5 milhões de pacientes com insuficiência cardíaca, e a mortalidade anual é em torno de $10 \%$, e entre $30 \%-40 \%$ para pacientes em classe funcional IV².

0 primeiro ecocardiograma da paciente, realizado no dia 30.7.2003, já mostrava doença cardíaca estrutural com disfunção sistólica acentuada, insuficiência mitral e tricúspide, além de volumoso derrame pericárdico.

O derrame pericárdico visto na ecocardiografia pode ser inicialmente interpretado como hidropericárdio, que ocorre em condições de intensa retenção hídrica, tal como na insuficiência cardíaca. Porém, essa apresentação é bem menos freqüente que o derrame pleural. Geralmente, o hidropericárdio não tem maiores conseqüências, a não ser quando muito volumoso, podendo levar a compressão de estruturas vizinhas, como o pulmão ${ }^{3}$. Em princípio não temos dados na história clínica inicial que possam sugerir outras causas para explicar o derrame pericárdico, como uma pericardite infecciosa, por vasculite, metabólica, neoplásica, traumática, ou por continuidade de estruturas (embolia pulmonar, infarto do miocárdio, aneurisma, dissecção da aorta e/ou pneumonia).

A doente apresentava também antecedente de evento isquêmico encefálico havia um ano, que sugere doença vascular aterosclerótica, a causa mais comum de acidente vascular cerebral, assim como dos fatores de risco para isso, como hipertensão arterial, diabete melito, tabagismo, arritmia cardíaca e doença valvar, todavia não relatados no caso clínico ${ }^{1}$.

Duas semanas após avaliação inicial, a paciente foi trazida ao hospital em síndrome de baixo débito cardíaco, caracterizada por depressão do status mental, hipoperfusão tecidual, extremidades frias e hipotensão ${ }^{4}$. Não tinha déficit motor aparente que sugerisse novo evento isquêmico cerebral e na ocasião apresentava sinais de insuficiência cardíaca direita, mas aparentemente sem congestão pulmonar, já que a semiologia desse sistema foi normal.

A principal causa de choque cardiogênico é o infarto agudo do miocárdio em conseqüência da perda de grande quantidade de músculo, geralmente mais de $40 \%$, ou perda menor de miocárdio em coração já doente 4 . A incidência do choque cardiogênico varia entre 5\% e 10\% dos infartos, com mortalidade extremamente elevada (70\%-100\%). Complicações mecânicas também podem levar a choque cardiogênico, como a ruptura de septo interventricular, ruptura ou disfunção de músculo papilar e ruptura de parede livre dos ventrículos ${ }^{5}$.

Outras causas de choque cardiogênico incluem infarto do ventrículo direito, taquicardias ou bradicardias refratárias e choque cardiogênico como parte da evolução final da disfunção miocárdica progressiva da doença de base, como doença isquêmica, idiopática, hipertrófica e restritiva. Deve também ser lembrado e analisado o status volêmico do paciente, já que a hipovolemia pode contribuir e agravar o choque cardiogênico ${ }^{4}$.

Discutindo e raciocinando sobre o choque cardiogênico, são esperados, além do baixo débito cardíaco, pressões de enchimento do ventrículo esquerdo elevadas. Todavia, no caso relatado, a paciente aparentemente não tinha congestão pulmonar. Das causas de choque cardiogênico apresentadas, o infarto do ventrículo direito, que geralmente acompanha o infarto de parede inferior, promove elevadas pressões de enchimento no coração direito (pressão venosa central e diastólica de ventrículo direito), enquanto as pressões esquerdas são normais ou discretamente elevadas, podendo justificar a semiologia pulmonar normal. Seguindo a mesma linha de pensamento, entrariam como diagnósticos diferenciais de choque com aumento de pressões direitas e pressões esquerdas normais o tamponamento pericárdico, a pericardite constritiva e a embolia pulmonar ${ }^{5}$.

Os eletrocardiogramas que mostram ritmo idioventricular e períodos de taquicardia ventricular não possibilitam a análise do segmento ST em qualquer derivação, nem sugerem um diagnóstico específico da doença de base. Como se sabe, as anormalidades na condução intraventricular e a dissociação atrioventricular podem precipitar ou intensificar a insuficiência cardíaca e o estado de choque ${ }^{6}$.

Os resultados laboratoriais mostram alteração da perfusão tecidual e da microcirculação, secundários ao baixo débito cardíaco - insuficiência renal com padrão pré-renal, aumento do lactato sérico e déficit de base encontrado na gasimetria.

A paciente, já na internação, apresentava vários fatores de mau prognóstico, como a idade superior a 65 anos, piora recente da classe funcional da insuficiência cardíaca, fatores hemodinâmicos, fatores laboratoriais (alteração da microcirculação, $\mathrm{Na}+$ sérico < $130 \mathrm{mEq} / \mathrm{l}$ ), necessidade de aminas vasoativas para manter pressão arterial e presença de taquicardia ventricular sustentada ou não ${ }^{7}$.

O ecocardiograma realizado no dia da internação não descreveu nenhuma alteração de contração segmentar 
que pudesse sugerir um choque cardiogênico secundário a infarto agudo do miocárdio, nem temos dados na história clínica que possam contribuir para esse diagnóstico. Evidenciava uma hipertrofia concêntrica de ventrículo esquerdo não descrita no primeiro ecocardiograma, mas que ajudaria a identificar a doença de base como, por exemplo, hipertrofia concêntrica do ventrículo esquerdo secundária a hipertensão arterial sistêmica. A valva aórtica era calcificada, mas sem estenose, afastando assim esse diagnóstico como causa de hipertrofia ventricular.

As miocardiopatias são classificadas como dilatadas, hipertróficas, restritivas, arritmogênica do ventrículo direito, ou ainda não-classificadas. Além disso, existem também as miocardiopatias específicas, relacionadas às suas etiologias, como miocardiopatias isquêmica, hipertensiva, valvar, inflamatória, metabólicas, periparto, por doenças sistêmicas, alcoólicas, por distrofias musculares, etc. ${ }^{8}$. Dentro do contexto do caso, afastase a maioria das causas específicas e também a miocardiopatia dilatada.

A miocardiopatia hipertrófica é encontrada em 0,2\% da população geral, na ausência de causas óbvias de hipertrofia como a hipertensão arterial e a estenose aórtica, freqüentemente com um predomínio septal e com ventrículo esquerdo não-dilatado. Pode ser obstrutiva (estenose subaórtica) ou não-obstrutiva, e a disfunção diastólica é bem maior que a sistólica ${ }^{9}$. 0 principal sintoma é a dispnéia em conseqüência da congestão pulmonar por aumento da pressão diastólica do ventrículo esquerdo. Desenvolve-se geralmente na adolescência, mas manifesta-se entre os 30-40 anos. Pode estar presente em idosos, com um início mais tardio, sendo nesses casos caracterizada por cavidades pequenas com discreta para moderada hipertrofia ventricular esquerda. Necropsias em octogenários têm mostrado que a miocardiopatia hipertrófica é bem mais freqüente do que parece. O principal achado ecocardiográfico é a hipertrofia ventricular esquerda, geralmente de caráter assimétrico (septal, anterolateral etc). Outros achados incluem a presença ou não de obstrução do fluxo de saída do ventrículo esquerdo, insuficiência mitral, átrio esquerdo dilatado, cavidade ventricular esquerda pequena, reduzida movimentação septal e anormalidades na função diastólica ${ }^{8,10}$.

A paciente em discussão poderia se encaixar no diagnóstico de miocardiopatia hipertrófica; porém, o argumento em contrário é que o ecocardiograma não mostrou acometimento assimétrico do ventrículo esquerdo e, além disso, essa doença geralmente não cursa com disfunção sistólica, a não ser em fases mais avançadas em que o ventrículo esquerdo começa a dilatar, o que não é o caso.

A miocardiopatia restritiva é forma pouco comum, e a sua principal característica é a anormal função diastólica do ventrículo esquerdo. As paredes do ventrículo esquerdo são extremamente rígidas, impedindo o seu enchimento, e a função sistólica, dependendo da causa, também pode estar acometida pela infiltração do miocárdio. Existem várias causas de miocardiopatia restritiva que podem ser divididas em causas miocárdicas (não-infiltrativas e infiltrativas) e endomiocárdicas. Dentre as miocárdicas não-infiltrativas, citamos a idiopática, a familiar, a secundária ao escleroderma e hipertrófica. Dentre as miocárdicas infiltrativas, citamos a amiloidose, a sarcoidose, a doença de Gaucher, a doença de Hurler, a hemocromatose, a glicogenose e a doença de Fabry. Dentre as endomiocárdicas, temos a endomiocardiofibrose, a síndrome carcinóide, as alterações por radiação e a cardiopatia de Loeffler ${ }^{8}$.

A amiloidose resulta no depósito de proteínas amilóides em diversos órgãos, incluindo o coração, ocorrendo por desordens hereditárias, inflamatórias e neoplásicas. Pode ser primária por depósito de imunoglobulinas de cadeia leve, como no caso do mieloma múltiplo, ou secundária a outras proteínas que não imunoglobulinas, associada a doenças inflamatórias, infecciosas e crônicas. Existe também a amiloidose familiar com caráter autossômico dominante e a amiloidose senil. No ecocardiograma verifica-se função sistólica normal nas fases iniciais, mas comprometida nos casos avançados, aumento da espessura das paredes ventricular esquerda e ventrículo de tamanho pequeno ou normal, átrio esquerdo dilatado, podendo ocorrer acometimento valvar. 0 derrame pericárdico é comum, mas raramente causa tamponamento. Pontos de hiper-refringência disseminados no músculo cardíaco e aparelhos valvares ${ }^{8,11,12}$ são encontrados.

Pelos exames laboratoriais, a paciente não apresentou indícios que pudessem sugerir mieloma múltiplo ou outra doença crônica (neoplásica ou inflamatória), já que não apresentou anemia nem inversão do padrão albumina/globulina. Porém, pelos dados apresentados acima, a amiloidose senil pode justificar os achados de disfunção sistólica e diastólica e o derrame pericárdico. Contudo, não foram descritos pontos de hiperrefrigência no ecocardiograma, o que não exclui o diagnóstico de amiloidose.

O ritmo idioventricular encontrado nesse caso, assim como ritmo juncional ou bloqueios atrioventriculares de graus variados, poderia ser justificado pelo depósito de substância amilóide no sistema de condução. Mas, como já mencionado, não é um achado específico de qualquer doença.

A cardiomiopatia restritiva idiopática é entidade rara, caracterizada por padrão restritivo, acometendo indivíduos de faixas etárias mais elevadas (quinta, sexta e sétima décadas de vida). Existem três formas: miocardiopatia restritiva pura; miocardiopatia restritiva hipertrófica; e dilatada restritiva. Nos achados de ecocardiografia encontramos aumento do átrio esquerdo, comprometimento da função diastólica, ventrículo esquerdo pequeno, derrame pericárdico e função sistólica geralmente preservada ${ }^{12}$. A paciente apresentava dados que podem sugerir miocardiopatia restritiva idiopática, principalmente em sua forma hipertrófica. 
Outras formas de miocardiopatia restritiva são mais raras ou seriam mais facilmente identificadas pelo ecocardiograma, como a endomiocardiofibrose.

Ainda analisando o segundo ecocardiograma, esse mostrou que o derrame pericárdico era discreto, excluindo tamponamento cardíaco, e não descreveu sinais sugestivos de pericardite constritiva que justificassem o choque apresentado pela paciente. O ventrículo direito era hipocinético com pressão de artéria pulmonar elevada, o que pode estar associado à doença de base ou ser de origem aguda, como no caso de uma embolia pulmonar, sendo essa forte hipótese para explicar o choque.

O terceiro ecocardiograma mostrou ventrículo esquerdo ainda menor. 0 átrio esquerdo, o átrio direito e o ventrículo direito encontravam-se bem aumentados; esse último apresentava aumento da espessura de suas paredes, assim como acentuada insuficiência tricúspide. Essas alterações sugerem fortemente o diagnóstico de embolia pulmonar em que se observam: dilatação e hipocinesia do ventrículo direito, hipertensão pulmonar, redução do diâmetro do ventrículo esquerdo; insuficiência tricúspide e tendência a hipertrofia ventricular direita.

A embolia pulmonar é a impactação de material dentro dos ramos do leito arterial pulmonar, sendo esse material, na maioria das vezes, trombo, mas ocorrendo também com células neoplásicas, gotículas de gordura, líquido aminiótico e substâncias exógenas injetadas na circulação ${ }^{13}$. A principal causa é a tromboembólica secundária a trombose venosa profunda. Nos Estados Unidos, a tromboembolia pulmonar (TEP) causa ou contribui para 50 mil mortes/ano, e $250 \mathrm{mil}$ hospitalizações por ano. A mortalidade na primeira hora é de $12 \%$, e atinge até $30 \%$ quando o diagnóstico não é prontamente realizado ${ }^{14}$. Vários são os fatores de risco para o TEP, como imobilização prolongada, trauma, queimaduras, cirurgias recentes, malignidade, obesidade, doença mieloproliferativa, gravidez, puerpério, deficiência de proteínas C e S e antitrombina III; contudo, no caso em discussão, devem ser ressaltadas a idade avançada e a insuficiência cardíaca congestiva ${ }^{13-16}$.

Existem várias formas de apresentação clínica da embolia pulmonar. Uma é o choque cardiogênico que ocorre por embolia pulmonar maciça ou uma embolia menor em um coração doente, provavelmente como aconteceu com essa paciente.

(Dr. George Paulo Cobe Fonseca)

Hipóteses diagnósticas $-1^{\circ}$ ) Choque cardiogênico por embolia pulmonar em paciente com miocardiopatia restritiva secundária a amiloidose senil; $2^{\circ}$ ) choque cardiogênico por embolia pulmonar em paciente com miocardiopatia restritiva idiopática - forma hipertrófica ou restritiva pura; $3^{\circ}$ ) choque cardiogênico por embolia pulmonar em paciente com miocardiopatia hipertrófica não-obstrutiva; $4^{\circ}$ ) choque cardiogênico por evolução da doença de base - miocardiopatia restritiva ou hipertrófica; $5^{\circ}$ ) choque cardiogênico por infarto agudo do ventrículo direito em paciente com miocardiopatia restritiva ou hipertrófica.

(Dr. George Paulo Cobe Fonseca)

\section{NeCROPSIA}

O coração pesou $578 \mathrm{~g}$, havendo dilatação moderada das quatro cavidades. As paredes cardíacas estavam espessadas e enrijecidas, tendo o miocárdio coloração castanho-amarelada e aspecto mosqueado. Presença de área irregular e discretamente deprimida na parede ântero-septal do ventrículo esquerdo, com componente hemorrágico, notando-se aí pequenos trombos aderidos ao endocárdio (fig. 2). O exame histológico do coração evidenciou deposição maciça de substância amilóide, principalmente no endocárdio e no interstício do miocárdio (fig. 3), notando-se também acometimento focal da microcirculação coronária (fig. 4). A lesão macroscópica descrita na parede ântero-septal do ventrículo esquerdo correspondia a múltiplos microinfartos em organização, com cerca de 7-14 dias de evolução, em meio a maciça deposição de substância amilóide (fig. 5). As artérias coronárias epicárdicas apresentavam lesões ateroscleróticas com obstruções máximas de 50\%-60\%, sem deposição aparente de substância amilóide. Os pulmões pesaram 700 g, notando-se deposição de substância amilóide nos septos alveolares (microcirculação), tromboembolia em organização em artérias de médio calibre e pequena área de infarto hemorrágico no lobo inferior esquerdo. Havia aterosclerose moderada da aorta e principais ramos, sendo detectada cicatriz de infarto cerebral antigo, acometendo núcleos da base à direita, com extensão para córtex frontal e áreas focais de infarto cicatrizado em ambos os rins. O fígado exibia degeneração hidrópica e esteatose de hepatócitos centrolobulares, com áreas de necrose hemorrágica e colestase, secundárias a choque cardiogênico prolongado.

(Dr. Luiz Alberto Benvenuti)

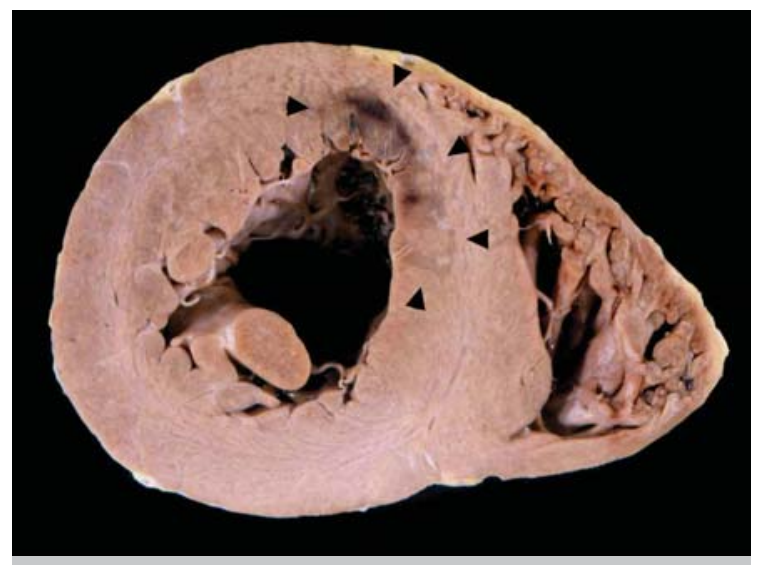

Fig. 2 - Macroscopia do coração - Corte transversal de ambos os ventrículos exibindo miocárdio de aspecto mosqueado e coloração castanho-amarelada. Note área discretamente deprimida, mais escurecida e hemorrágica na parede ântero-septal do ventrículo esquerdo, que corresponde às áreas de microinfarto em organização (cabeças de seta). 


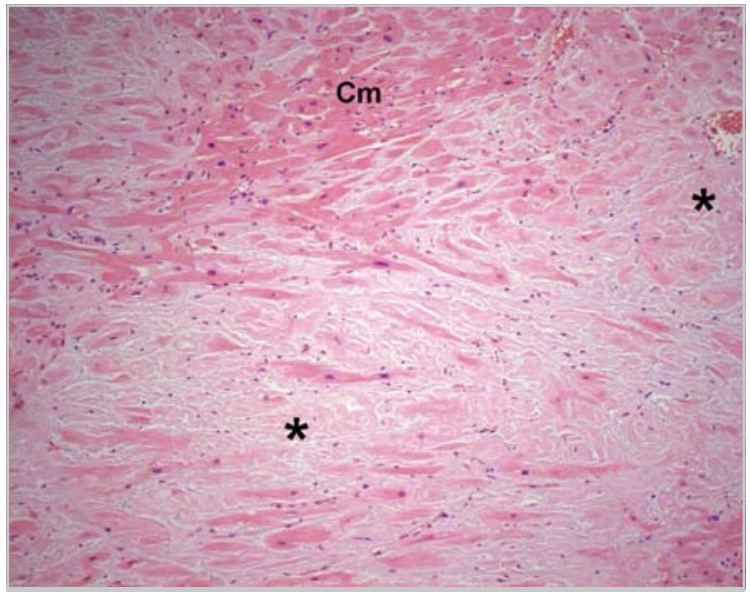

Fig. 3 - Depósitos intramiocárdicos de substância amilóide - corte histológico do miocárdio evidenciando maciça deposição de substância amilóide no interstício (asteriscos), ilhando grupos de cardiomiócitos (Cm). Hematoxilina-eosina, fotografado com objetiva de $10 \times$.

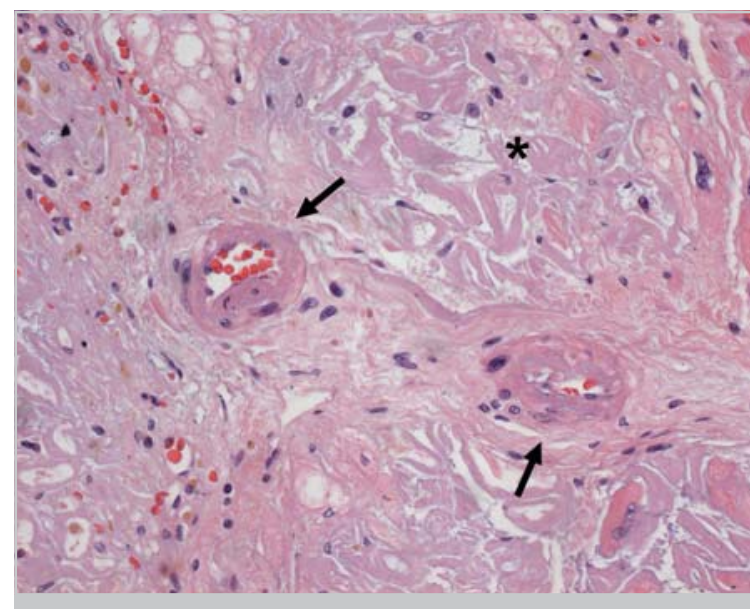

Fig. 4 - Depósito amilóide arteriolar. Em meio a maciça deposição de substância amilóide no miocárdio (asterisco), nota-se também deposição na parede de arteríolas (setas). Hematoxilina-eosina, fotografado com objetiva de $40 \times$.

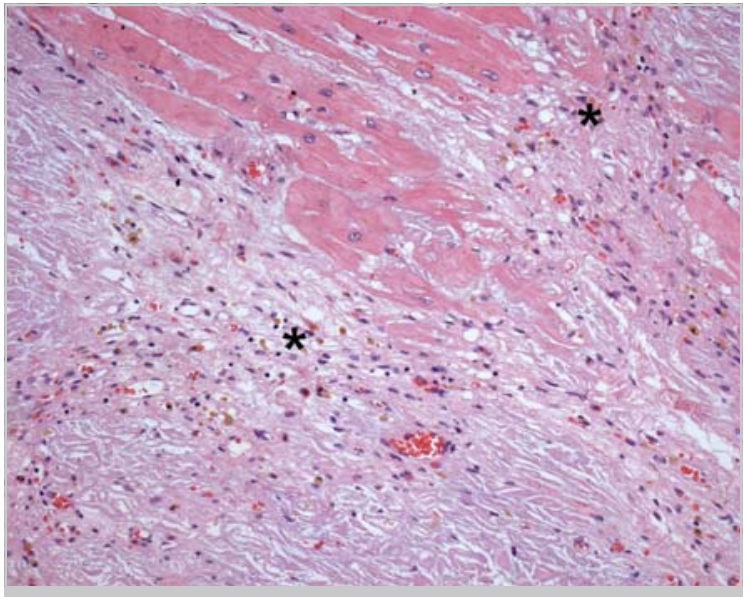

Fig. 5 - Microinfartos em parede ântero-septal do ventrículo esquerdo - nas vizinhanças de áreas de deposição intersticial de substância amilóide, notam-se microinfartos em organização, caracterizados pela presença de tecido de granulação (asteriscos). Hematoxilina-eosina, fotografado com objetiva de $20 \times$.
Diagnósticos anatomopatológicos - Amiloidose cardiovascular senil; microinfartos em organização, localizados na parede ântero-septal do ventrículo esquerdo; aterosclerose sistêmica com infartos cicatrizados no cérebro e nos rins; tromboembolia pulmonar com pequeno infarto hemorrágico; alterações secundárias a choque cardiogênico prolongado (causa terminal do óbito).

(Dr. Luiz Alberto Benvenuti)

\section{Comentários}

Trata-se de paciente idosa, do sexo feminino, apresentando amiloidose cardiovascular senil com deposição maciça de substância amilóide no miocárdio, acarretando espessamento e enrijecimento das paredes cardíacas. Apresentava ainda aterosclerose sistêmica, com infartos antigos no cérebro e nos rins; entretanto, a aterosclerose das artérias coronárias epicárdicas não era exuberante, havendo obstruções máximas de 50\%$60 \%$. Evoluiu com insuficiência cardíaca progressiva, microinfartos em parede ântero-septal do ventrículo esquerdo, tromboembolia pulmonar e choque cardiogênico prolongado, que culminou com o óbito.

0 coração é freqüentemente acometido na amiloidose, em qualquer de suas formas. No período de 1889 a 1977, foram identificados 223 pacientes portadores de amiloidose que faleceram e foram submetidos a necropsia no Johns Hopkins Hospital.

Nesses, o coração esteve acometido em 108 casos (48\%), sendo 63 casos de amiloidose senil, 26 de amiloidose primária, 5 de amiloidose secundária, 11 associadas ao mieloma múltiplo, e 3 de amiloidose familiar. Na amiloidose, a disfunção cardíaca pode manifestar-se como insuficiência cardíaca congestiva, tanto sistólica como diastólica (restritiva), valvopatia ou arritmia. Na vigência de comprometimento cardíaco, a substância amilóide pode estar depositada em qualquer dos três folhetos (endocárdio, miocárdio e pericárdio), sempre no espaço extracelular, sendo freqüente o acometimento da microcirculação coronária.

A amiloidose cardíaca tem sido reconhecida como uma das causa de infarto do miocárdio desacompanhado de lesões obstrutivas significativas das coronárias epicárdicas ${ }^{17}$. Em tais casos, a deposição maciça de substância amilóide na microcirculação, mais comum nos casos de amiloidose primária, estaria associada ao desenvolvimento das lesões isquêmicas ${ }^{18-20}$. Podemos especular também que a maciça deposição de substância amilóide no interstício do miocárdio poderia acarretar compressão extrínseca da microcirculação e dos próprios cardiomiócitos, que se tornariam então necróticos ${ }^{18}$.

No presente caso, a amiloidose era cardiovascular senil e havia lesões ateroscleróticas das artérias coronárias epicárdicas, apesar da ausência de obstruções críticas. 0 encontro de áreas de infarto cicatrizado no cérebro e nos rins atesta a presença de aterosclerose sistêmica. Dessa 
forma, cremos que as áreas de microinfarto do miocárdio deveram-se à combinação dos fatores isquêmicos anteriormente citados, quer sejam o comprometimento aterosclerótico das artérias coronárias epicárdicas, a maciça deposição de substância amilóide no interstício quer o comprometimento direto da microcirculação.

(Dr. Luiz Alberto Benvenuti)

\section{REFERÊNCIAS}

1. Carbajal EV, Deedwania PC. Congestive Heart Failure. In: Crawford $\mathrm{MH}$. Current Diagnosis and Treatment in Cardiology. $2^{\text {nd }}$ Ed. New York: McGraw-Hill, 2003: 217-49.

2. Revisão da II Diretriz da Sociedade Brasileira de Cardiologia para o Diagnóstico e Tratamento da Insuficiência Cardíaca. Arq Bras Cardiol. 2002; 79(Supl. IV): 1-30.

3. Spodick DH. Pericardial Diseases. In: Braunwald E, Zipes DP, Libby P. Heart Disease. $6^{\text {th }}$ Ed. Philadelphia, Pennsylvania: W.B. Saunders Co., 2001: 1823-76.

4. Timm C, Cardiogenic Shock. In: Crawford MH. Current Diagnosis and Treatment in Cardiology. $2^{\text {nd }}$ Ed. New York: McGraw-Hill, 2003: 88-96.

5. Antman EM, Braunwald E. Acute Myocardial Infarction. In: Braunwald E, Zipes DP, Libby P. Heart Disease. $6^{\text {th }}$ Ed. Philadelphia, Pennsylvania: W.B. Saunders Co. 2001:1114-1231.

6. Gilvertz MM, Colucci WS, Braunwald E. Clinical Aspects of Heart Failure: High-output Failure; Pulmonary Edema. In: Braunwald E, Zipes DP, Libby P. Heart Disease. $6^{\text {th }}$ Ed. Philadelphia, Pennsylvania: W.B. Saunders Co., 2001: 534-561.

7. Aminoff MJ. Nervous System. In: Tierney LM, Mcphee SJ, Papadakis MA. Current Medical Diagnosis and Treatment. $4^{\text {th }}$ Ed. New York: McGraw-Hill, 2001: 969-1027.

8. Wynne J, Braunwald E. The Cardiomyopathies and Myocarditides. In: Braunwald E, Zipes DP, Libby P. Heart Disease. $6^{\text {th }}$ Ed. Philadelphia, Pennsylvania: W.B. Saunders Co., 2001: 1751-1806.

9. Nishimura RA, Holmes DR. Hipertrophic obstructive cardiomyopathy. N Eng J Med. 2004; 350: 1320-7.

10. Shah P. Hipertrophic Cardiomyopathies. In Crawford MH. Current Diagnosis and Treatment in Cardiology. $2^{\text {nd }}$ Ed. New York: McGraw-Hill, 2003: 179-87.

11. Carroll JD, Crawford MH. Restrictive Cardiomyopathies. In Crawford $\mathrm{MH}$. Current Diagnosis and Treatment in Cardiology. $2^{\text {nd }}$ Ed. New York: McGraw-Hill, 2003: 188-95.

12. Kushwaha SS, Fallon JT, Fuster V. Restrictive cardiomiopathy. N Eng J Méd. 1997; 336: 267-77.

13. Baruzzi ACA, Knobel E. Tromboembolismo Pulmonar. In: Knobel E. Condutas no Paciente Grave. $2^{\text {nd }}$ Ed. São Paulo: Atheneu, 1999: 197210.

14. Gilvertz MM, Colucci WS, Braunwald E. Clinical Aspects of Heart Failure: High-output Failure; Pulmonary Edema. In: Braunwald E, Zipes DP, Libby P. Heart Disease. $6^{\text {nd }}$ Ed. Philadelphia, Pennsylvania: W.B. Saunders Co., 2001: 534-61.

15. Goldhaber SZ. Pulmonary Embolism. In: Braunwald E, Zipes DP, Libby P. Heart Disease. $6^{\text {nd }}$ Ed. Philadelphia, Pennsylvania: W.B. Saunders Co., 2001: 1886-907.

16. Goldhaber SZ. Pulmonary Embolic Disease. In Crawford MH. Current Diagnosis and Treatment in Cardiology. $2^{\text {nd }}$ Ed. New York: McGraw-Hill, 2003: 365-81

17. Smith RRL, Hutchins GM. Ischemic heart disease secondary to amyloidosis of intramyocardial arteries. Am J Cardiol. 1979; 44: 413-17.

18. Barbour DJ, Roberts WC. Frequency of acute and healed myocardial infarcts in fatal cardiac amyloidosis. Am J Cardiol 1988; 62: 1134-5.

19. Neben-Wittich MA, Wittich CM, Mueller OS, Larson DR, Gertz MA, Edwards WD. Obstructive intramural coronary amyloidosis and myocardial ischemia are common in primary amyloidosis. Am J Med. 2005; 118: 1287.

20. Cantwell RV, Aviles RJ, Bjornsson J, Wright RS, Freeman WK, Oh JK, et al. Cardiac amyloidosis presenting with elevation of cardiac troponin I and angina pectoris. Clin Cardiol. 2002; 25: 33-7. 